\title{
MENINGIOMAS ANTERIORES E ANTERO-LATERAIS DO FORAME MAGNO
}

\author{
Osvaldo Inácio de Tella Jr', Manoel Antonio de Paiva Neto², \\ Paulo Henrique Aguiar', Marco Antonio Herculano ${ }^{4}$
}

\begin{abstract}
RESUMO - Relatamos nossa experiência com 11 pacientes port a d o res de meningiomas do forame magno, oito cranioespinhais e três espinocraniais. A média de idade foi 50,8 anos, o sintoma mais comum foi cefaléia occipital com duração média de 18,6 meses. Os principais achados neurológicos foram tetraparesia e comprometimento dos nervos cranianos baixos. O tratamento foi cirúrgico, sempre com exposição da a rtéria vertebral em sua entrada na dura-máter da fossa posterior e ressecção de parte do côndilo occipital apenas em três casos. Ressecção total foi possível em sete pacientes e parcial nos demais, devido às aderências a vasos e nervos. O prognóstico esteve relacionado com as condições neurológicas pré-operatórias.
\end{abstract}

PALAVRAS-CHAVE: meningioma, forame magno, artéria vertebral.

\begin{abstract}
Anterior and lateral foramen magnum meningiomas
ABSTRACT - We report our experience with 11 cases of foramen magnum meningiomas, eight originating inside the posterior fossa and three in the caudal region. The mean age of the patients was 50.8 years and the main complaint was cervical headache for at least 18.6 months and at the neurological examination, tetrapa resisand deficit of the lower cranial nerves were very often observed. All patients were submitted to surgical treatment, always with exposition of the vertebral art e ry at the entry zone in the duramater of the posterior fossa, with partial removal of the occipital condyle in only three cases. Total resection was obtained in seven patients and partial removal in the other four due to adherences to vessels and nerves. The prognostic was related to the neurological condition before surgery.
\end{abstract}

KEY WORDS: meningioma, foramen magnum, vertebral artery.

A implantação e origem dos meningiomas, na região do forame magno, é verificada na dura-máter da junção crânio-vertebral. Os limites dessa região são: anterior, do terço inferior do clivo à margem superior do corpo do axis; lateralmente é delimitada pelo tubérculo jugular e margem superior da lâmina do axis; posterior, da porção anterior do osso occipital ao processo espinhal do axis ${ }^{1,2}$. Meningiomas anteriores do forame magno são aqueles com base de implantação anterior no plano coronal, correspondente ao primeiro ligamento denteado inferiormente e nervos bulbares superiormente. Devem ser distinguidos dos demais devido a diferenças de apresentação clínica, manejo cirúrgico e complicações ${ }^{3}$. Ainda podem ser classificados como espinocraniais quando tem predomínio de crescimento caudal e cranioespinhais quando o predomínio de crescimento é cranial ${ }^{4}$.
Os meningiomas da transição crânio-cervical perfazem de 1,2 a 3,2\% do total de meningiomas intracranianos e intra-raquidianos $^{1,5}$. Na série de Olivecrona (apud Castellano, Ruggiero-1953) chegam a 4\% dos meningiomas de fossa posterior, sendo precedidos por meningiomas da porção posterior do osso petroso (42\%); tentório (30\%); clivo (11\%) e convexidade cerebelar $(10 \%)^{5}$.

A p resentamos a experiência no tratamento cirúrgico de 11 pacientes portadores de meningioma do forame magno.

\section{MÉTODO}

Entre 1996 e 2005, 11 pacientes portadores de meningiomas do forame magno for am submetidos aressecção cirúrgca no Hospital Professor Edmundo Vasconcellos e no Hospital São Paulo. Todos os pacientes foram submetidos

1P rofessor Adjunto Livre Docente da Disciplina de Neurociru rgia da Universidade Federal de São Paulo (UNIFESP) e Médico do Hospital Professor Edmundo Vasconcelos (HPEV), São Paulo, Brasil; ${ }^{2}$ Médico Neurociru rgião do Hospital São Paulo e Médico do HPEV; ${ }^{3}$ Neurocirurgião Assistente da Divisão de Neurocirurgia do Hospital das Clínicas da Universidade de São Paulo, Brasil; ${ }^{4}$ Professor Adjunto da Disciplina de Neurocirurgia da Faculdade de Medicina de Jundiaí e médico do HPEV.

Recebido 25 Agosto 2005, recebido na forma final 16 Janeiro 2006. Aceito 7 Março 2006.

Dr. Osvaldo Inácio de Tella Jr - Rua São Paulo Antigo 145 / Bloco F / Apto 11 - 05864-010 São Paulo SP - Brasil. E-mail: detella@globo.com 


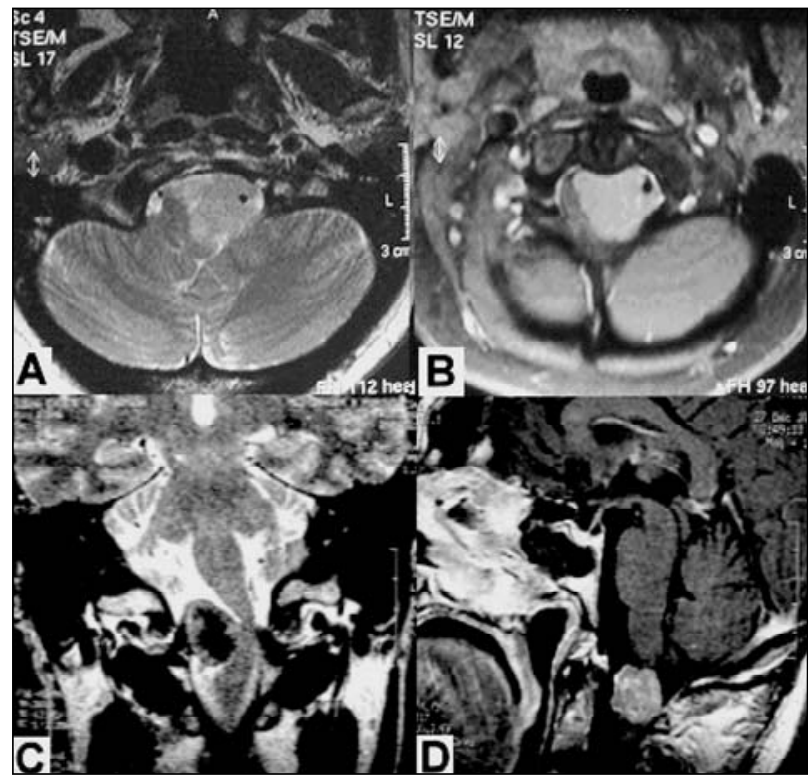

Fig 1. RM transição cranio-vertebral seqüência $T 2$ a direita (B) e após injeção de gadolínio a esquerda (A) mostrando menin gioma cranioespinal anterior do forame magno. RM corte sagi tal após injeção de gadolínio a direita (D) e corte coronal em $T 2$ a esquerda (C), mostrando meningioma anterior de forame magno de localização espino-cranial.

a ressonância magnética (RM) pré-operatória que mostrava presença de processo expansivo na região anterior e/ou anterolateral do forame magno (Fig 1). Sete pacientes eram do sexo feminino e quatro do sexo masculino com média de idade 50,8 anos (38-74 anos).

A queixa mais comum foi cefaléia nucal (nove casos), seguida de tetraparesia espástica (oito casos), a duração média das queixas foi 18,6 meses. Outros sintomas encontrados foram: dificuldade de deglutição em dois casos, ataxia em um paciente e hemiatrofia de língua também em um caso.

Todos foram submetidos a ressecção neurocirúrgica por via suboccipital posterolateral com ressecção parcial do côndilo occipital quando julgado necessário, mas sempre com exposição da área de entrada da artéria vertebral na dura-máter da transição occipitocer vical. Ressecção tota I do tumor foi conseguida em sete casos e confirmada por tomografia computadorizada (TC) e ou RM pós-operatória. Em três casos a ressecção foi subtotal devido a sua aderência a vasos ou a nervos cranianos e em um parcial devido a extensão bilateral do tumor. Todos os casos foram classificados como meningeoma grau I OMS.

\section{RESULTADOS}

No pós-operatório imediato, três pacientes evoluíram com broncopneumonia aspirativa secundária a paresia de nervos cranianos baixos. Fístula liqüórica pós-operatória foi observada em um caso. O acompanhamento, que variou de seis meses a nove anos, evidenciou nove pacientes com melhora ou estabilidade dos déficites neurológicos comprovados pela escala de Kamofsky. Uma paciente portadora de tumor com extensão bilateral foi submetida a ressecção parcial e apresentou piora do déficit motor no pós-operatório. Uma paciente que apresentava tetraparesia grave no pré-operatório foi submetida a ressecção subtotal e também evoluiu com piora do déficit motor, não obtendo melhora da deglutição.

Não foi observada recorrência/recrescimento tumoral sintomático na presente série até o presente momento.

\section{DISCUSSÃO}

A primeira ressecção de tumor do forame magno foi relatada por Hallopeau, em 1874'. Em estudo de 102 casos de tumores extra-axiais benignos do forame magno, operados de 1924 à 1982, Meyer et al. encontraram 78 meningiomas, 23 neuro $\mathrm{f} i \mathrm{~b}$ romas e 1 teratoma ${ }^{6}$. Entretanto, na série da Sociedade de Neurocirurgia de Língua Francesa, em 1993, foram analisados 230 casos de tumores extramedulares desta região, entre os quais 106 eram meningiomas, 49 neurinomas, 28 cordomas, 32 tumores ósseos (19 primários e 13 metastáticos) e 15 casos considerados como tumores incomuns (quatro melanomas, três hemangioblastomas, três tumores dermóides ou epidermóides, dois ependimomas, um cavernoma, um angiomiolipoma e um granuloma de colesterol). A média de idade dos pacientes era 47 anos, com predominância do sexo feminino de 1,5 / 1, similar aos dados observados em nossa casuística ${ }^{1}$.

O intervalo médio de aparecimento dos sintomas e o diagnóstico do tumor é cerca de dois anos e três meses, variando de seis a 96 meses. Os meningiomas de grande volume situados na face ventral do forame magno cursam com deterioração neurológica mais grave e rapidamente progressiva ${ }^{7}$. Sintoma bastante referido e geralmente precoce na evolução é a cefaléia occipitocervical, que piora com flexão da cabeça e manobra de Valsalva ${ }^{8}$. O exame neurológico pode revelar alterações de tratos longos como: hiperreflexia, sinal de Babinski e marcha paraparética-espástica; alterações da sensibilidade proprioceptiva e/ou superficiais e, quase sempre, envolvimento do XI primeiro nervo craniano e, raramente, do nervo hipoglosso unilateral, como em um de nossos pacientes. É recomendável o exame dos músculos trapézio e esternocleidomatóide a procura de sinais de atrofia. Atrofia e/ou fasciculações de língua podem ocorrer. Aproximadamente $40 \%$ dos pacientes podem ter exame neurológico normal ${ }^{1,9}$. Em $81 \%$ de nossos casos a cefaléia estava presente e em $72 \%$, algum grau de tetraparesia. 
Quadros de tetraparesia e paraparesia espástica com reflexos vivos e ou exaltados de evolução progressiva devem ser avaliados sempre por meio de RM da coluna cervical e transição craniocervical, sendo o exame de escolha para o diagnóstico destas lesões. As imagens na seqüência $T 2$ devem ser cuidadosamente estudadas, verificando-se a presença de plano de aracnóide entre tumor e tecido cerebral e a presença de edema neural que sugerem invasão pial pelo tumor. Nestes casos uma ressecção subtotal será necessária com intuito de presenação de função neurológica. O diagnóstico diferencial deve ser feito com doenças neurológicas tais como: esclerose lateral amiotrófica, esclerose múltipla, espondilose cenical, tumores intramedulares, seringomielia, síndrome do túnel do carpo, e mesmo hidrocefalia de pressão normal ${ }^{10,11}$.

A TC reconstruída em três dimensões, e com uso de contraste iodado, permite verificar a relação do tumor com o tubérculo jugular bem como sua posição em relação ao côndilo occipital ${ }^{12-14}$. A angiografia digital ou angioressonância auxilia na análise da nutrição vascular do tumor e sua relação com estruturas de drenagem venosa (Fig 2). A artéria vertebral englobada e estreitada pelo tumor pode significar invasão de sua adventícia por tecido tumoral, tornando perigosa a ressecção total. A embolização de tumores nesta região é discutível ${ }^{12-14}$.

A cirurgia de meningiomas nesta região está semp re indicada, pois o prognóstico pós-operatório está relacionado com os déficits pré-existentes ${ }^{13}$. A abordagem extremolateral foi desenvolvida para facilitar remoção de tumores do clivo inferior e forame magno anterior. $O$ conceito de remover osso suficiente $e$ prover via de acesso ao tumor, sem retração de estruturas nervosas consiste na ressecção da borda inferior do forame magno até o côndilo occipital e também da lâmina ou hemilâmina posterior do atlas, podendo ser acrescida a ressecção parcial ou completa do côndilo occipital (via transcondilar) ${ }^{15-17}$. Alguns autores acreditam que esta via não é necessária, sendo estes tumores passíveis de ressecção utilizandose a via suboccipital posterior convencional, evitandose assim uma abordagem mais demorada e com risco maior de lesão de estruturas vasculonervosas como a artéria vertebra| ${ }^{8,18,19}$.

Em nossos casos sempre procuramos expor a artéria vertebral deslocando-a de seu sulco na porção superior da lâmina do atlas e individualizando sua entrada na dura-máter. A ressecção parcial do côndilo occipital foi realizada em três casos de nossa casuística

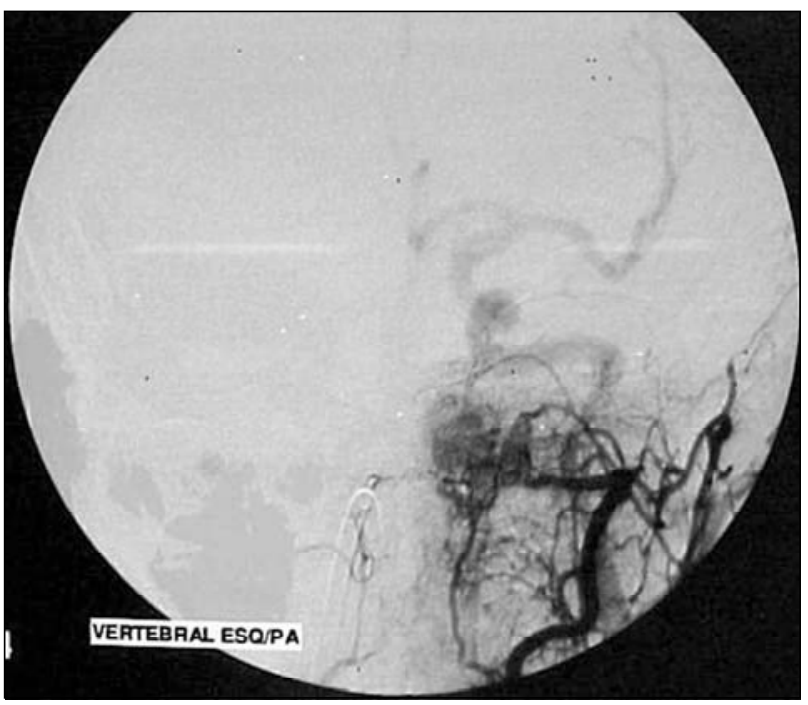

Fig 2. Angiografia digital vertebral esquerda mostrando o apor te vascular e drenagem venosa do meningioma.

em que o tumor crescia bilateralmente. Em oito pacientes foi utilizada a via suboccipital lateral sem ressecção do côndilo, mas com exposição da artéria vertebral e boa exposição tumoral já que nestes pacientes o crescimento tumoral deslocou o tronco cerebral em direção contralateral. A principal causa de ressecção subtotal dos tumores foi a aderência do tumor aos vasos e nervos cranianos, e não devida ao tipo de exposição. Também foi descrita a via trans-oral para abordagem destes tumores, quando situados entre as duas artérias vertebrais, mas esta técnica não tem sido utilizada pelo risco de fístula liquórica ${ }^{20}$.

Sempre que possível, a vascularização do tumor deve ser interrompida por meio da coagulação da sua inserção. $O$ esvaziamento tumoral, independente do acesso cirúrgico deve ser realizado por meio de aspirador ultrassônico e coagulação bipolar. A artéria espinal anterior está normalmente aderida a porção posterior do tumor e deve ser dissecada no espaço subaracnoideo.

O XI nervo craniano tem trajeto longitudinal descendente e se adere à margem lateral do tumor. A a rtéria vertebral é freqüentemente deslocada para trás e lateralmente, aumentando a distância entre as duas artérias vertebrais, pode ser totalmente envolvida pelo tumor no seu ponto de entrada na fossa posterior, fato observado em três casos de nossa série. A artéria cerebelar póstero-inferior é normalmente deslocada para cima. A artéria vertebral é transposta de acordo com seu deslocamento e grau de envolvimento pelo tumor $1-4,13,18,19,21$.

Monitoração eletrofisiológica intra-operatória po- 
de ajudar o neurocirurgião na preservação de funções n e u rológicas. O uso de potencial evocado somatossensitivo permite o estudo das vias ascendentes. A eletroneuromiografia da língua e dos músculos esternocleidomastoide e trapézio permitem avaliação dos nervos hipoglosso e acessório espinhal ${ }^{3,4,22}$. Em nenhum caso de nossa série foi utilizada monitoração per-operatória.

A ressecção micro-cirúrgica total é o tratamento de escolha para os pacientes portadores de meningiomas do forame magno. Todavia, em pacientes com a ressecção tumoral parcial ou subtotal (tumores residuais) e em tumores recidivados a radiociru rgia estereotáxica é importante opção complementar. Por outrolado, nos pacientes idosos sem condições clínicas para uma cirurgia de grande porte, ou em pacientes não idosos sem condições clínicas para a cirurgia, a radiocirurgia deve ser cogitada como tratamento alternativo ${ }^{23}$.

Revisão da literatura, realizada por Boulton e Cusimano mostrou índice de bons resultados cirúrgicos de 70 a $90 \%$ nas séries mais recentes. A mortalidade cirúrgica variou de 0 a $15 \%{ }^{8}$. Prognóstico pior é observado em pacientes com alterações neurológicas graves no pré-operatório, como observado em um caso de nossa casuística. Não houve mortalidade na nossa série. Dois pacientes (18\%) se achavam neurologicamente piores em relação ao pré-operatório, seis meses após a cirurgia.

As seqüelas pós-operatórias descritas na literatura incluem piora da paresia motora, perda de função dos pares cranianos baixos com piora da disfagia, tosse e aspiração de alimentos e secreções, observadas principalmente se não há déficit no pré-operatório. $\mathrm{O}$ tratamento requer fisioterapia, traqueostomia e anastomose traqueo-esofágica1 $1-4,8,24$.

Meningeoma do forame magno é doença de freqüência incomum que, apesar de localização em região de anatomia complexa, pode ser retirado com baixa morbidade, utilizando-se técnica microcirúrgica adequada ${ }^{25}$.

\section{REFERÊNCIAS}

1. George B, Lot G, Velut S, Gelbert F, Mourier KL. [French Language Society of Neurosurgery. $44^{\text {th }}$ Annual Congress. Brussels, 8-12 June 1993. Tumors of the foramen magnum]. Neurochirurgie 1993;39(Suppl 1):S81-S89.
2. GeorgeB, Lot $G$, Boissonnet $H$. Meningioma of the foramen magnum: a series of 40 cases. Surg Neurol 1997;47:371-379.

3. Arnautovic KI, Al-Mefty O, Husain M. Ventral foramen magnum meningiomas. J Neurosurg 2000;92:71-80.

4. Al-Mefty O. Meningiomas of the craniovertebral junction. In: Al Mefty O (ed): Operative atlas of meningiomas. Philadelphia: Lippincott. Raven, 1998:349-382.

5. Castellano F, Ruggiero G. Meningioma of posterior fossa. Acta Radiol 1953;(Suppl 104):S1-S157.

6. Meyer FB, Ebersold MJ, Reese DF. Benign tumors of the foramen magnum. J Neurosurg 1984;61:136-142.

7. Guidetti B, Spallone A. Benign extramedullary tumors of the foramen magnum. Surg Neurol 1980;13:9-17.

8. Boulton MR, Cusimano MD. Foramen magnum meningiomas: concepts, classifications and nuances. Neurosurg Focus 2003;14:6, article10.

9. Chono $\mathrm{Y}$, Abe H, Iwasaki $Y$, et al. Transoral anterior approach to foramen magnum meningioma: a case report and review. No Shinkei Geka 1985;13:109-114.

10. Sawaya RA. Foramen magnum meningioma presenting as amyotrophic lateral sclerosis. Neurosurg Rev 1998;21:277-280.

11. Yasuoka S, Okazaki H, Daube JR, MacCarty CS. Foramen magnum tumors; analysis of 57 cases of benign extramedullary tumors. J Neurosurg 1978;49:828-838.

12. Lee BC, Deck MD, Kneeland JB, Cahill PT. MR imaging of the craniocervical junction. Am J Neuroradiol 1985;6:209-213.

13. Ojemann RG. Foramen magnum meningioma. In Tatter SB (ed). Clinical neurosurgery. New York: Saunders, 1992:321-383.

14. Kanai M, Kawano K, Uehara S. A case of foramen magnum meningioma in which case enhanced three-dimensional CT scan was valuable for preoperative evaluation of the surgical approach. No Shinkei Geka 1997;25:641-645.

15. Sen CN, Sekhar LN. An extreme lateral approach to intradural lesions of the cervical spine and foramen magnum. Neurosurgery 1990;27: 197-204.

16. Spetzler RF, Graham TW. The far lateral approach to the inferior clivus and upper cervical region: technical note. Barrow Neurol Inst Q 1990;6: 35-38.

17. Bertalanffy H, Seeger W. The dorsolateral, suboccipital, transcondylar a p proach to the lower clivus and anterior portion of craniocervical junction. Neurosurgery 1991;29:815-821.

18. Goel A, Desai K, Muzumdar D. Surgery on anterior forâmen magnun meningiomas using a conventional posterior suboccipital approach: a report on a experience with cases. Neurosurgery 2001;49:102-115.

19. Nanda A, Vincent DA, Vannenredy PSS, Chanda A. Far-lateral approach to intradural lesions of the foramen magnum without resection of occipital condyle. J Neurosurg 2002:96:302-309.

20. Miller E, Crocka rd HA. Transoral transclival removal of anteriorly placed meningiomas at the foramen magnum. Neuro su rgery 1987;20: 966-968.

21. Rhoton AL Jr, de Oliveira E. Micro s u rical anatomy of the region of foramen magnum. In Wilkins RH, Rengachary SS (eds). Neurosurgery update. I: diagnosis, operative technique, and neuro-oncology. New York: McGraw Hill, 1990:434-461.

22. Wagner W, Peghini-Halbig L, Mäurer JC, Perneczky A . Intraoperative SEP monitoring in neurosurgery around the brain stem and cervical spinal cord: differential recording of subcortical components. J Neurosurg 1994;1:213-220.

23. Muthukumar N, Kondziolka D, Lundsford, Flickinger JC. Stereotatic radiosurgery for foramen magnum meningiomas. Surg Neurol 1999:51:268-273.

24. Hongladaom T, Lindeman RC. Tracheo-esophageal anastomosis in treatment of chronic aspiration pneumonitis. Arch Phys Med Rehabil 1987;59:246-247.

25. Samii M, Klekamp J, Carvalho G. Surgical results for meningiomas of the craniocervical junction. Neurosurgery 1996;39:1086-1095. 